\title{
Preparation of Biodegradable Silk Fibroin/Alginate Blend Films for Controlled Release of Antimicrobial Drugs
}

\author{
Yaowalak Srisuwan and Yodthong Baimark \\ Biodegradable Polymers Research Unit, Department of Chemistry and Center of Excellence for Innovation in Chemistry, \\ Faculty of Science, Mahasarakham University, Mahasarakham 44150, Thailand
}

Correspondence should be addressed to Yodthong Baimark; yodthong.b@msu.ac.th

Received 6 May 2013; Revised 29 July 2013; Accepted 15 August 2013

Academic Editor: Jie Dai

Copyright ( 2013 Y. Srisuwan and Y. Baimark. This is an open access article distributed under the Creative Commons Attribution License, which permits unrestricted use, distribution, and reproduction in any medium, provided the original work is properly cited.

Silk fibroin (SF)/alginate blend films have been prepared for controlled release of tetracycline hydrochloride, an antimicrobial model drug. The blend films were analysed by Fourier transform infrared (FTIR) spectroscopy, scanning electron microscopy (SEM), and UV-vis spectroscopy. The functional groups of the SF/alginate blends were monitored from their FTIR spectra. The homogeneity of the blend films was observed from SEM images. The dissolution and film transparency of the blend films depended on the SF/alginate blend ratio. The in vitro drug release profile of the blend films was determined by plotting the cumulative drug release versus time. It was found that the drug release significantly decreased as the SF/alginate blend ratio increased. The results demonstrated that the SF/alginate blend films should be a useful controlled-release delivery system for water-soluble drugs.

\section{Introduction}

Controlled-release drug delivery is a term referring to drug delivery in response to time. Prolonged release, sustained release, and delayed release are terms used to identify drug delivery systems that are designed to deliver a precise amount of a drug at a preprogrammed rate in order to achieve the drug level necessary for treatment after administration of a single dose [1]. Controlled-release drug delivery not only prolongs the action, thus reducing the frequency of drug administration, but also attempts to maintain drug levels within the therapeutic window to avoid potentially hazardous peaks in drug concentration following injection or ingestion and to maximise therapeutic efficiency. Various types of controlled-release drug delivery system have been prepared such as films, particles, gels, and fibres. Polymeric materials have been widely investigated for use as matrices of drug delivery systems. The drug delivery systems obtained from biodegradable polymers have the advantage over nonbiodegradable polymers in that the removal of these devices at the completion of therapy is not required. The biodegradable polymers consist of natural type such as silk fibroin, chitosan, starch, and alginate and synthetic type such as polylactic acid, polyglycolic acid, and poly( $\varepsilon$ caprolactone). Usually, the natural biodegradable polymers are less expensive.

Silk fibroin (SF) obtained from Bombyx mori silkworm is a protein polymer [2]. SF has been widely studied for use in tissue engineering [3] and drug delivery applications $[4,5]$. SF in film, fibre, tube, gel, and particle forms has been prepared for these purposes. SF has two conformational forms: water-soluble (random coil) and water-insoluble ( $\beta$ sheet). The conformational transition of SF from the random coil form to the $\beta$-sheet form can be done by treatment with alcohol, heat, cross-linker, and polymeric blending [2] .

Alginates are natural polysaccharides that have many uses in biomedical and pharmaceutical applications due to their low toxicity, biocompatibility, and biodegradability $[6,7]$. Alginate-based devices have been widely used as drug carriers for controlled release due to their low immunogenicity and mucoadhesive properties [8-10]. However, alginate is easily dissolved in aqueous media. Cross-linking of alginate with $\mathrm{Ca}^{2+}$ ions has been used to reduce their swelling and dissolution [8].

In the present study, SF/alginate blend films containing a model drug were prepared by the solvent evaporation 
<smiles>CC(C)NCC(=O)N[C@@H](CO)C(=O)NCC(=O)N[C@@H](C)C(=O)N[C@@H](C)C(=O)NCC1CCC1</smiles>

(a)

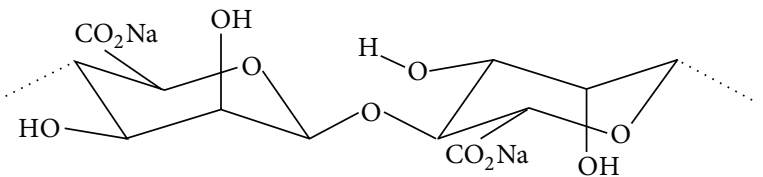

(b)<smiles>CN(C)[C@H]1C(O)=C(C(N)=O)C(=O)[C@]2(O)C(O)=C3C(=O)c4c(O)cccc4[C@](C)(O)[C@H]3C[C@H]12</smiles>

(c)

FIgURE 1: Chemical structures of (a) silk fibroin, (b) alginate, and (c) tetracycline hydrochloride.

method. The influence of SF/alginate blend ratio on film characteristics and drug release behaviour was determined. Tetracycline hydrochloride was chosen as an antimicrobial model drug due to its good water solubility. SF, alginate, and drug were blended in an aqueous medium before film casting. Tetracycline hydrochloride has been shown to be clinically effective in the treatment of periodontal infection [11]. The chemical structures of SF, alginate, and tetracycline hydrochloride are illustrated in Figure 1.

\section{Materials and Methods}

2.1. Materials. Silk fibroin (SF) aqueous solution was prepared as follows. Briefly, cocoons of B. mori gifted from the Silk Innovation Centre, Mahasarakham University, Thailand, were degummed by boiling twice in $0.5 \%$ (w/v) $\mathrm{Na}_{2} \mathrm{CO}_{3}$ solution at $95^{\circ} \mathrm{C}$ for $1 \mathrm{~h}$, followed by rinsing thoroughly with distilled water to remove sericin before drying at room temperature [12]. Degummed SF fibres were dissolved in the ternary solvent system, $\mathrm{CaCl}_{2}$-ethanol-water (molar ratio $=1: 2: 8)$, by stirring at $85^{\circ} \mathrm{C}$ for $2 \mathrm{~h}$. The resultant $\mathrm{SF}$ solution was then dialysed in a dialysis tube (molecular weight cutoff $6,000-8,000 \mathrm{Da}$ ) for 3 days in distilled water. The distilled water was changed every day. The SF solution was filtered before adjusting to $1 \%(\mathrm{w} / \mathrm{v})$ with distilled water. Sodium alginate $\left(\left(\mathrm{C}_{6} \mathrm{H}_{7} \mathrm{O}_{6} \mathrm{Na}\right)_{n}\right.$, pharmaceutical grade $)$ was purchased from Carlo Erba. The intrinsic viscosity of the sodium alginate measured in $0.1 \mathrm{M} \mathrm{NaCl}$ water solution at $25^{\circ} \mathrm{C}$ using an Ubbelohde viscometer was $850 \mathrm{~mL} / \mathrm{g}$. Tetracycline hydrochloride $\left(\mathrm{C}_{22} \mathrm{H}_{24} \mathrm{~N}_{2} \mathrm{O}_{8} \cdot \mathrm{HCl}, 99 \%\right)$ was used as a water-soluble model drug and purchased from Sigma. The $1 \%(\mathrm{w} / \mathrm{v})$ alginate solution was prepared using distilled water as the solvent.

2.2. Preparation of Drug-Loaded Blend Films. SF/alginate blend films containing the model drug were prepared by the solvent evaporation method. Appropriate volumes of $1 \%(\mathrm{w} / \mathrm{v})$ SF and $1 \%(\mathrm{w} / \mathrm{v})$ alginate solutions were blended together by magnetic stirring for $30 \mathrm{~min}$. The total volume of the blend solution was kept constant at $20 \mathrm{~mL}$. The model drug ( $2 \mathrm{mg}$ ) was completely dissolved in the blend solution

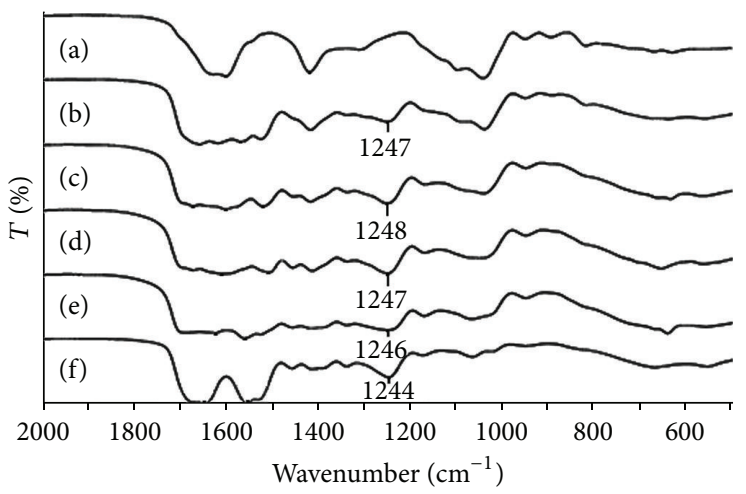

FIGURE 2: FTIR spectra of drug-loaded blend films with SF/alginate blend ratios of (a) 0/1, (b) 1/1, (c) 2/1, (d) 4/1, (e) 8/1, and (f) 1/0 (w/w).

before film casting on polystyrene dishes. SF/alginate blend ratios of $8 / 1,4 / 1,2 / 1$, and 1/1 (w/w) were investigated. Plain SF and alginate films were also prepared for comparison. The (SF + alginate)/drug ratio was kept constant at 100/1 (w/w). The blend solution was dried at $40^{\circ} \mathrm{C}$ for $48 \mathrm{~h}$. The obtained films were kept in a desiccator before property characterisation and drug release testing.

2.3. Characterisation of Drug-Loaded Blend Films. Chemical functional groups of the blend films were analysed by Fourier transform infrared (FTIR) spectroscopy using a PerkinElmer Spectrum GX FTIR spectrophotometer. A resolution of $4 \mathrm{~cm}^{-1}$ and 32 scans were employed. The morphology of the blend films was determined by scanning electron microscopy (SEM) using a JEOL JSM-6460LV SEM. The blend film samples were coated with gold to enhance the surface conductivity before the scan. Film transparency was measured from the percentage of transmittance at $660 \mathrm{~nm}$ $\left(\% T_{660}\right)[13]$ by UV-vis spectrophotometry using a PerkinElmer Lambda 25 UV-vis spectrophotometer. Dissolution of the films $(\sim 10 \mathrm{mg})$ was tested in $2 \mathrm{~mL}$ of distilled water at $37^{\circ} \mathrm{C}$ for $24 \mathrm{~h}$ under shaking. The remaining film was 


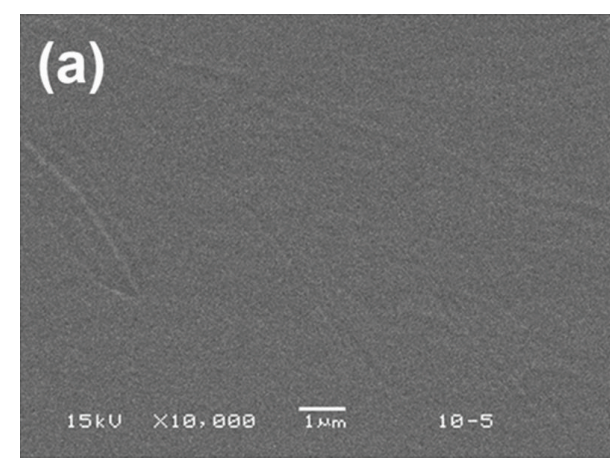

(a)

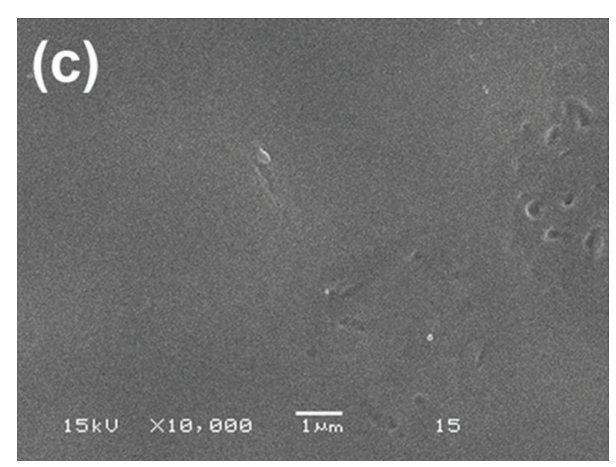

(c)

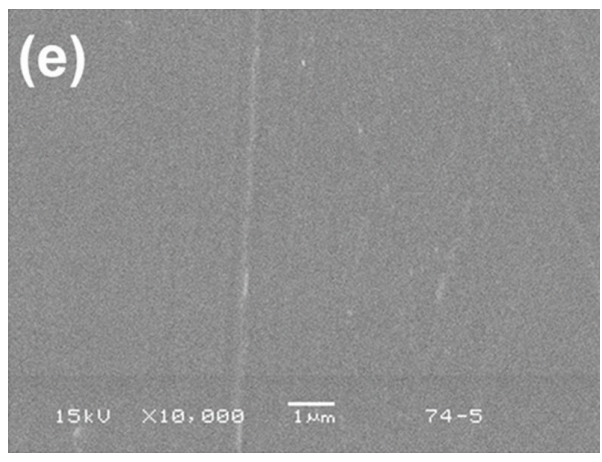

(e)

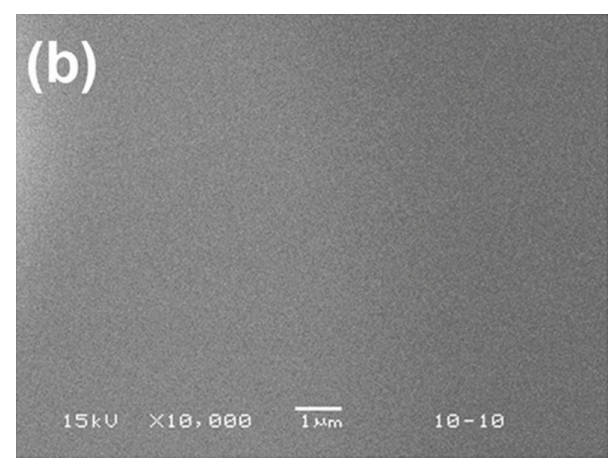

(b)

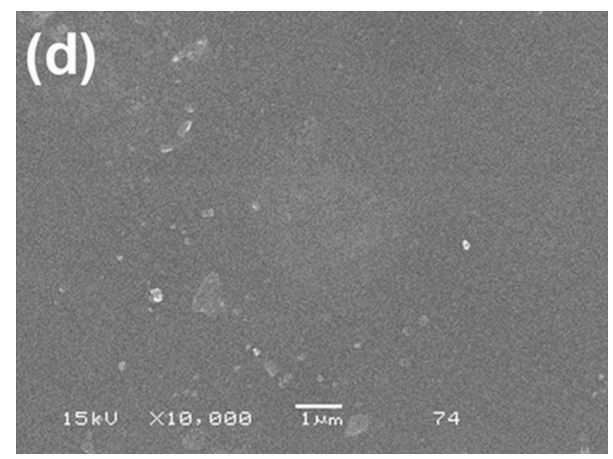

(d)

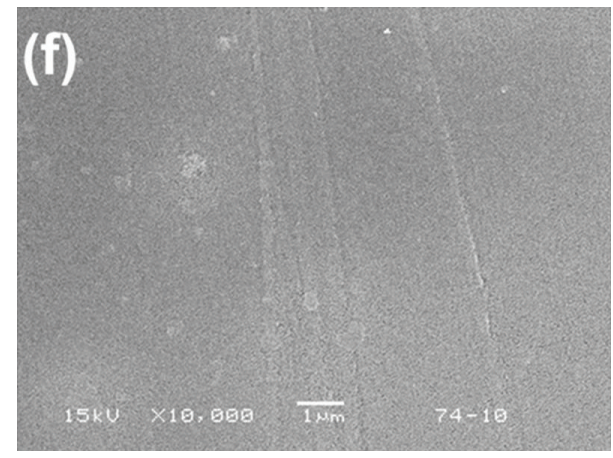

(f)

FIGURE 3: SEM micrographs of surfaces of drug-loaded blend films with SF/alginate blend ratios of (a) 0/1, (b) 1/1, (c) 2/1, (d) 4/1, (e) 8/1, and (f) $1 / 0(\mathrm{w} / \mathrm{w})$. All bars $=1 \mu \mathrm{m}$.

collected by centrifugation and freeze-dried before weighing. The dissolution was calculated from the following:

$$
\text { Dissolution }(\%)=\left[\frac{(A-B)}{A}\right] \times 100 \text {, }
$$

where $A$ and $B$ are the weights of the starting and remaining films, respectively.

2.4. In Vitro Drug-Release Test. An in vitro drug-release test was performed in $1 \mathrm{mM}$ PBS buffer $(\mathrm{pH} 7.4)$ at $37^{\circ} \mathrm{C}$ under shaking. The drug-loaded blend films $(\sim 10 \mathrm{mg})$ were immersed in $2 \mathrm{~mL}$ of buffer. At the desired time, $1 \mathrm{~mL}$ of drug solution was collected after centrifugation at 5,000 rpm for $5 \mathrm{~min}$. The $1 \mathrm{~mL}$ of fresh buffer was then replaced. The drug release was determined by UV-vis spectrophotometry at $\lambda_{\max }=360 \mathrm{~nm}$ and compared to a standard curve of drug solution. Cumulative drug release was calculated in terms of the ratio of the cumulative mass of the released drug at a given time against the initial drug loading in the film sample. In vitro drug release tests were run in triplicate $(n=3)$.

\section{Results and Discussion}

3.1. FTIR of Blend Films. FTIR spectra were used to determine the chemical functional groups and molecular interactions between SF and alginate as shown in Figure 2. The FTIR spectrum of the alginate film in Figure 2(a) shows two distinctive absorption bands at around $1600 \mathrm{~cm}^{-1}$ and $1418 \mathrm{~cm}^{-1}$ relative to both asymmetric and symmetric $\mathrm{C}=\mathrm{O}$ stretching absorptions of the carboxylic acid salts [14]. The absorption band at $1000-1200 \mathrm{~cm}^{-1}$ is attributed to the 


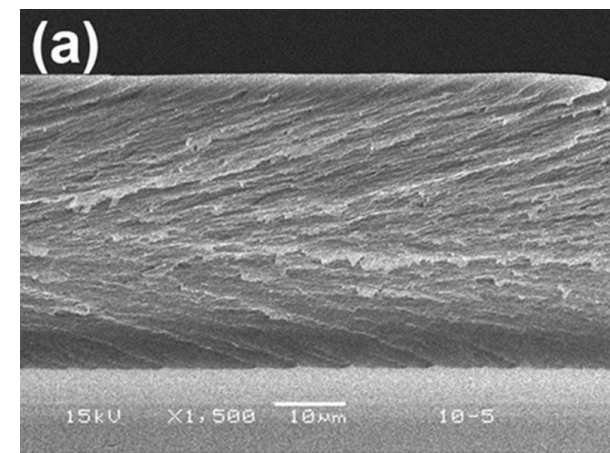

(a)

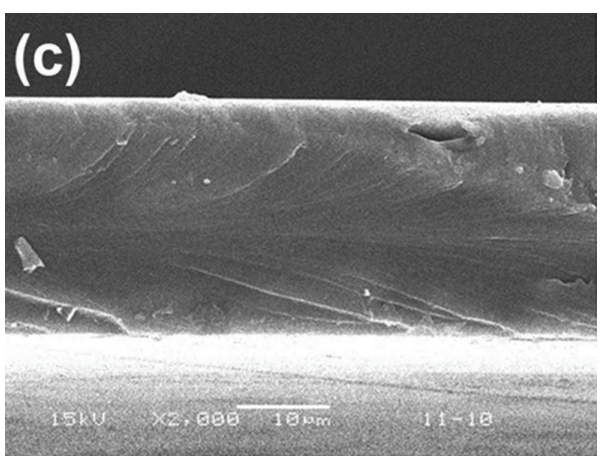

(c)



(e)



(b)

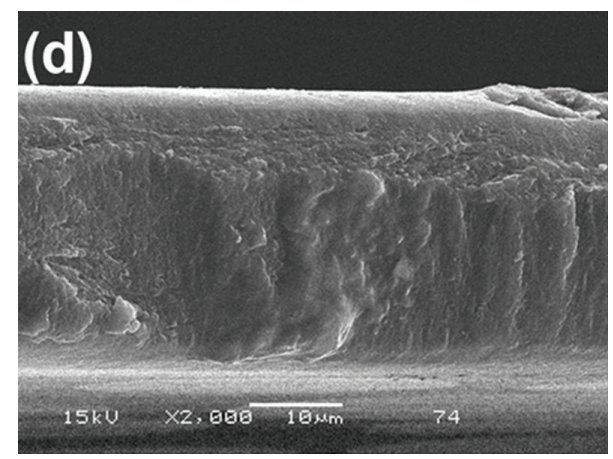

(d)

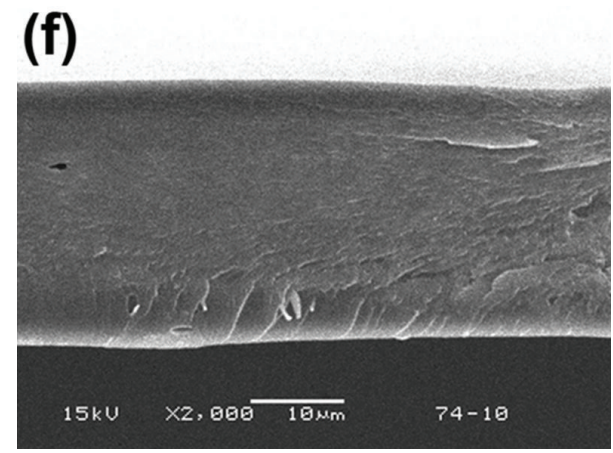

(f)

Figure 4: SEM micrographs of cross-sections of drug-loaded blend films with SF/alginate blend ratios of (a) 0/1, (b) 1/1, (c) 2/1, (d) 4/1, (e) $8 / 1$, and (f) $1 / 0(\mathrm{w} / \mathrm{w})$. All bars $=10 \mu \mathrm{m}$.

characteristic saccharide absorptions of polysaccharides [15, 16]. The FTIR spectrum of the SF film in Figure 2(f) exhibited absorption bands at $1641 \mathrm{~cm}^{-1}$ (amide I), $1551 \mathrm{~cm}^{-1}$ (amide II), and $1244 \mathrm{~cm}^{-1}$ (amide III), which are assigned to the predominantly random coil form $[17,18]$. The characteristic absorption bands of both SF and alginate characters were found in the FTIR spectra of all the blend films. As would be expected, the band intensities of the alginate's distinctive absorptions $(\mathrm{C}=\mathrm{O}$ symmetric stretching and saccharide bands) significantly decreased as the SF/alginate blend ratio increased as shown in Figures 2(b)-2(e). The band intensities of SF amide III seem similar to those of the $8 / 1,4 / 1$, and $2 / 1$ (w/w) SF/alginate blend films. This is due to the fact that SF is dominant in these blend films. However, the band intensity of SF amide III significantly decreased as the SF/alginate blend ratio decreased to $1 / 1(\mathrm{w} / \mathrm{w})$, as shown in Figure 2(b).
The band intensities of SF and alginate depended on the blend ratio. In addition, the amide III bands of the SF component shifted to a higher wavenumber after blending with alginate, suggesting interactions between the SF and alginate in the blend films. These interactions may be the hydrogen bonds between the carbonyl groups of SF and hydroxyl groups of alginate.

3.2. Morphology and Transparency of Blend Films. Figures 3 and 4 show SEM micrographs of the film surface and cross-section, respectively. Film thicknesses observed from Figure 4 were in the range of $40-60 \mu \mathrm{m}$. It can be seen that both film surfaces and cross-sections of the SF, alginate, and their blend films were homogeneous. For the blend films, phase separation was not observed in all of the blend ratios. This is due to the fact that both the SF and alginate are 


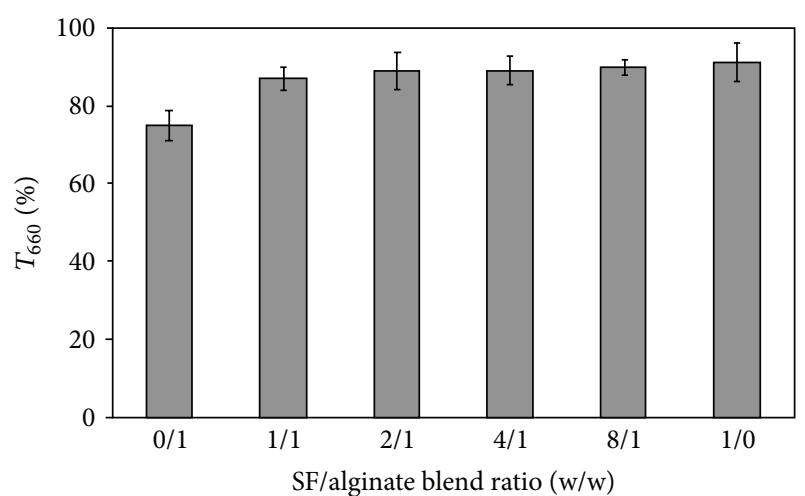

FIgURE 5: $T_{660}$ of drug-loaded blend films with different SF/alginate blend ratios.

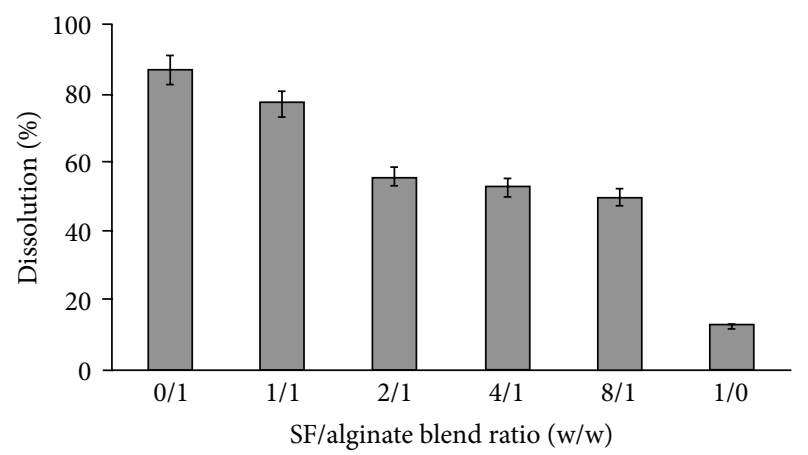

FIGURE 6: Dissolution of drug-loaded blend films with different $\mathrm{SF} /$ alginate blend ratios.

hydrophilic polymers and are miscible in aqueous media before film casting.

The SF and blend films were highly transparent, whereas the alginate film was slightly opaque. Film transparencies were compared from their $\% T_{660}$ values. A higher $\% T_{660}$ value indicates higher film transparency. Figure 5 shows the $\% T_{660}$ of the SF, alginate, and their blend films. The $\% T_{660}$ values of the SF and alginate films were $91 \%$ and $75 \%$, respectively; that is, the SF film showed higher transparency than that of the alginate film. The film transparency of the blend films slightly decreased as the SF/alginate blend ratio decreased. The film transparency results supported the fact that the blend films consisted of different SF/alginate blend ratios as according to the FTIR results.

3.3. Dissolution of Blend Films. Figure 6 shows the dissolution behaviour in distilled water of SF, alginate, and their blend films. The dissolutions of SF and alginate films were $13 \%$ and $84 \%$, respectively. The low dissolution of the SF film may be due to the SF containing strong hydrogen bonds. The dissolution of the blend films was lower than that of the alginate film and decreased as the SF/alginate blend ratio increased. This result indicates that the dissolution of the blend films can be controlled with the SF/alginate blend ratio.

3.4. Drug Release of Blend Films. Figure 7 shows the in vitro drug release profiles of the film samples in phosphate buffer

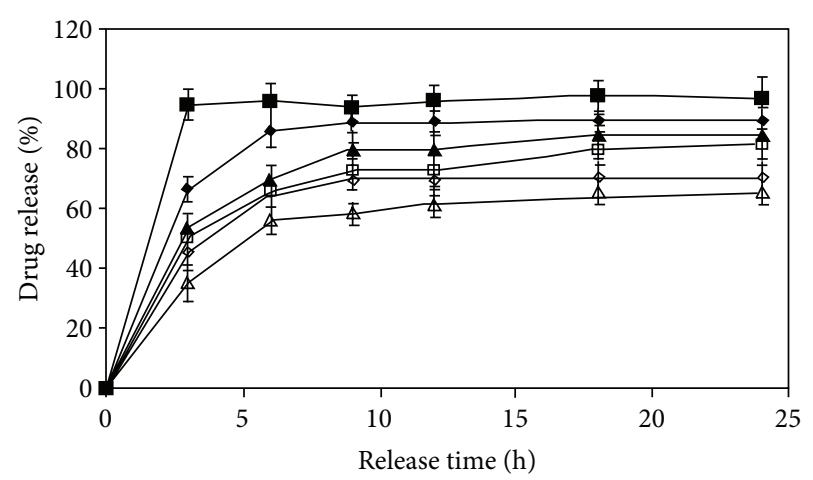

FIGURE 7: In vitro drug release profiles of blend films with


and $(\triangle) 1 / 0(\mathrm{w} / \mathrm{w})$.

solution ( $\mathrm{pH}=7.4$ ) at $37^{\circ} \mathrm{C}$ for $24 \mathrm{~h}$. In the case of the alginate film (SF/alginate ratio $=0 / 1)$, around $95 \%$ of the loaded drug was released within $3 \mathrm{~h}$, due to the fast dissolution of the alginate film matrix. The SF film $(\mathrm{SF} /$ alginate ratio $=1 / 0)$ showed the lowest drug release, which might be due to the lowest dissolution of the SF film matrix. For all the SF/alginate blend films, a sustained drug release can be observed. For $\mathrm{SF} /$ alginate blend ratios of $0 / 1,1 / 1,2 / 1,4 / 1,8 / 1$, and $1 / 0(\mathrm{w} / \mathrm{w})$, the drug release levels at $24 \mathrm{~h}$ were $95 \%, 90 \%, 85 \%, 82 \%$, $71 \%$, and $64 \%$, respectively. This result revealed that the drug release steadily decreased as the $\mathrm{SF} /$ alginate blend ratio increased. The lower dissolution of SF provided a decreased drug release level of the blend films as previously described.

\section{Conclusions}

In this research, the drug-loaded SF/alginate blend films were prepared by the simple solvent evaporation method. The drug, SF, and alginate were blended in an aqueous medium before film casting. SEM images reveal that the drug-loaded blend films for all SF/alginate blend ratios are homogeneous. The transparency and dissolution of the blend films strongly depend on SF/alginate blend ratio. The drug release profiles can be adjusted by varying the SF/alginate blend ratio. These $\mathrm{SF} /$ alginate blend films are potential as controlled-release delivery media for water-soluble drugs.

\section{Acknowledgments}

The authors gratefully acknowledge Mahasarakham University for financial support. The Center of Excellence for Innovation in Chemistry (PERCH-CIC), Office of the Higher Education Commission, Ministry of Education, Thailand, is also acknowledged.

\section{References}

[1] V. Kumar, S. K. Prajapati, G. C. Soni, M. Singh, and N. Kumar, "Sustained release matrix type drug delivery system: a review," World Journal of Pharmacy and Pharmaceutical Sciences, vol. 1, pp. 934-960, 2012. 
[2] G. H. Altman, F. Diaz, C. Jakuba et al., "Silk-based biomaterials," Biomaterials, vol. 24, no. 3, pp. 401-416, 2003.

[3] L. Meinel, R. Fajardo, S. Hofmann et al., "Silk implants for the healing of critical size bone defects," Bone, vol. 37, no. 5, pp. 688698, 2005.

[4] A. S. Lammel, X. Hu, S.-H. Park, D. L. Kaplan, and T. R. Scheibel, "Controlling silk fibroin particle features for drug delivery," Biomaterials, vol. 31, no. 16, pp. 4583-4591, 2010.

[5] E. M. Pritchard, C. Szybala, D. Boison, and D. L. Kaplan, "Silk fibroin encapsulated powder reservoirs for sustained release of adenosine," Journal of Controlled Release, vol. 144, no. 2, pp. 159$167,2010$.

[6] K. Y. Lee and D. J. Mooney, "Alginate: properties and biomedical applications," Progress in Polymer Science, vol. 37, no. 1, pp. 106126, 2012.

[7] H. Wu, C. Liao, Q. Jiao, Z. Wang, W. Cheng, and Y. Wan, "Fabrication of core-shell microspheres using alginate and chitosanpolycaprolactone for controlled release of vascular endothelial growth factor," Reactive Functional Polymers, vol. 72, pp. 427437, 2012.

[8] A. Shilpa, S. S. Agrawal, and A. R. Ray, "Controlled delivery of drugs from alginate matrix," Journal of Macromolecular Science C, vol. 43, no. 2, pp. 187-221, 2003.

[9] J. K. Oh, D. I. Lee, and J. M. Park, "Biopolymer-based microgels/nanogels for drug delivery applications," Progress in Polymer Science, vol. 34, no. 12, pp. 1261-1282, 2009.

[10] C. A. García-González, M. Alnaief, and I. Smirnova, "Polysaccharide-based aerogels-promising biodegradable carriers for drug delivery systems," Carbohydrate Polymers, vol. 86, no. 4, pp. 1425-1438, 2011.

[11] S. P. Vyas, V. Sihorkar, and V. Mishra, "Controlled and targeted drug delivery strategies towards intraperiodontal pocket diseases," Journal of Clinical Pharmacy and Therapeutics, vol. 25, no. 1, pp. 21-42, 2000.

[12] J. Zhou, C. Cao, X. Ma, L. Hu, L. Chen, and C. Wang, "In vitro and in vivo degradation behavior of aqueous-derived electrospun silk fibroin scaffolds," Polymer Degradation and Stability, vol. 95, no. 9, pp. 1679-1685, 2010.

[13] Y. Baimark, N. Niamsa, N. Morakot, J. Threeprom, and Y. Srisuwan, "Preparation and morphology study of biodegradable chitosan/methoxy poly(ethylene glycol)-b-poly( $\varepsilon$-caprolactone) nanocomposite films," International Journal of Polymer Analysis and Characterization, vol. 12, no. 6, pp. 457-467, 2007.

[14] A. Joshi, R. Keerthiprasad, R. D. Jayant, and R. Srivastava, "Nano-in-micro alginate based hybrid particles," Carbohydrate Polymers, vol. 81, no. 4, pp. 790-798, 2010.

[15] R. C. Mundargi, N. B. Shelke, A. P. Rokhade, S. A. Patil, and T. M. Aminabhavi, "Formulation and in-vitro evaluation of novel starch-based tableted microspheres for controlled release of ampicillin," Carbohydrate Polymers, vol. 71, no. 1, pp. 42-53, 2008.

[16] A. Roy, J. Bajpai, and A. K. Bajpai, "Dynamics of controlled release of chlorpyrifos from swelling and eroding biopolymeric microspheres of calcium alginate and starch," Carbohydrate Polymers, vol. 76, no. 2, pp. 222-231, 2009.

[17] Y. Srisuwan, P. Srihanam, and Y. Baimark, "Preparation of silk fibroin microspheres and its application to protein adsorption," Journal of Macromolecular Science A, vol. 46, no. 5, pp. 521-525, 2009.

[18] Y. Hu, Q. Zhang, R. You, L. Wang, and M. Li, "The relationship between secondary structure and biodegradation behavior of silk fibroin scaffolds," Advances in Materials Science and Engineering, vol. 2012, Article ID 185905, 5 pages, 2012. 

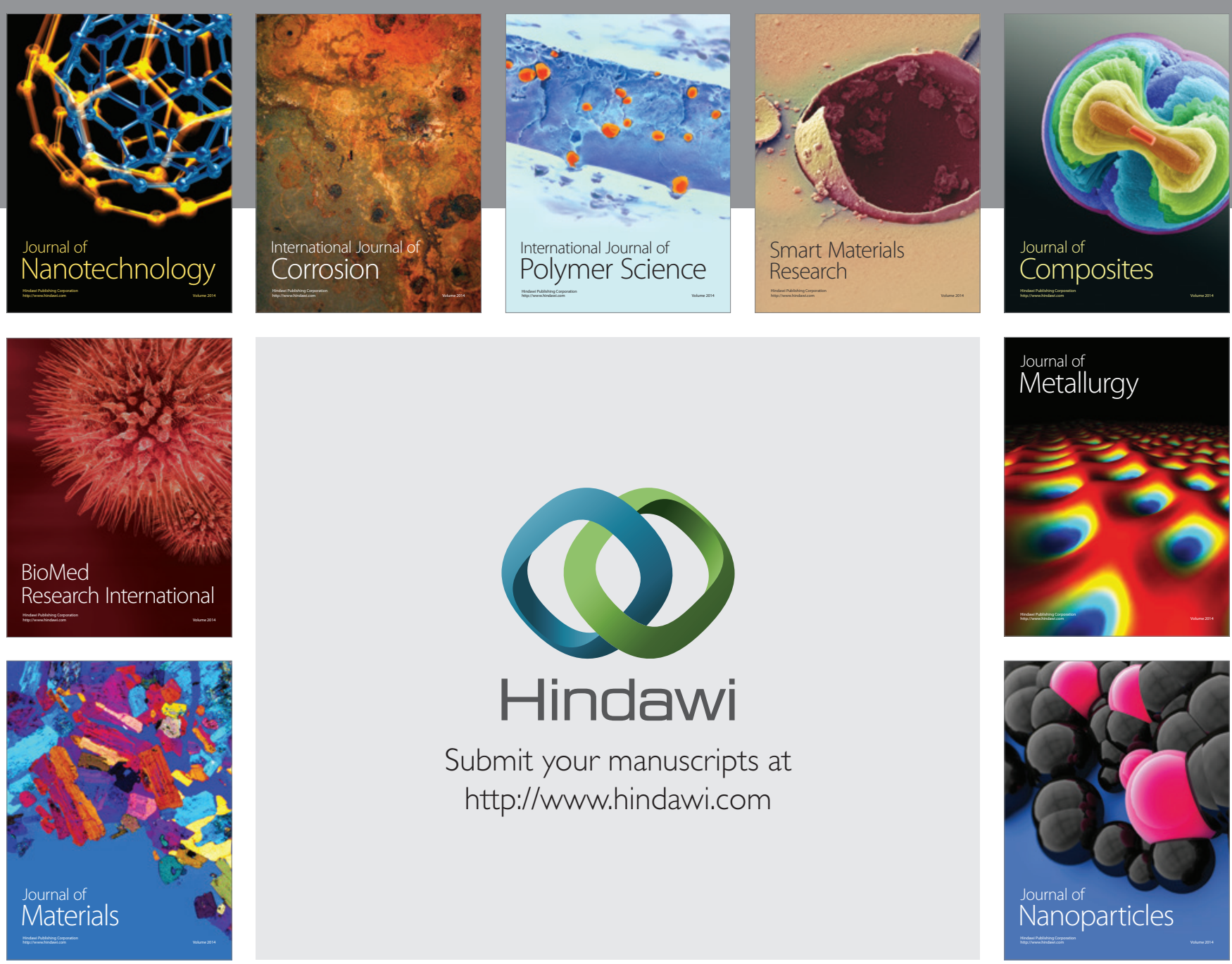

Submit your manuscripts at http://www.hindawi.com
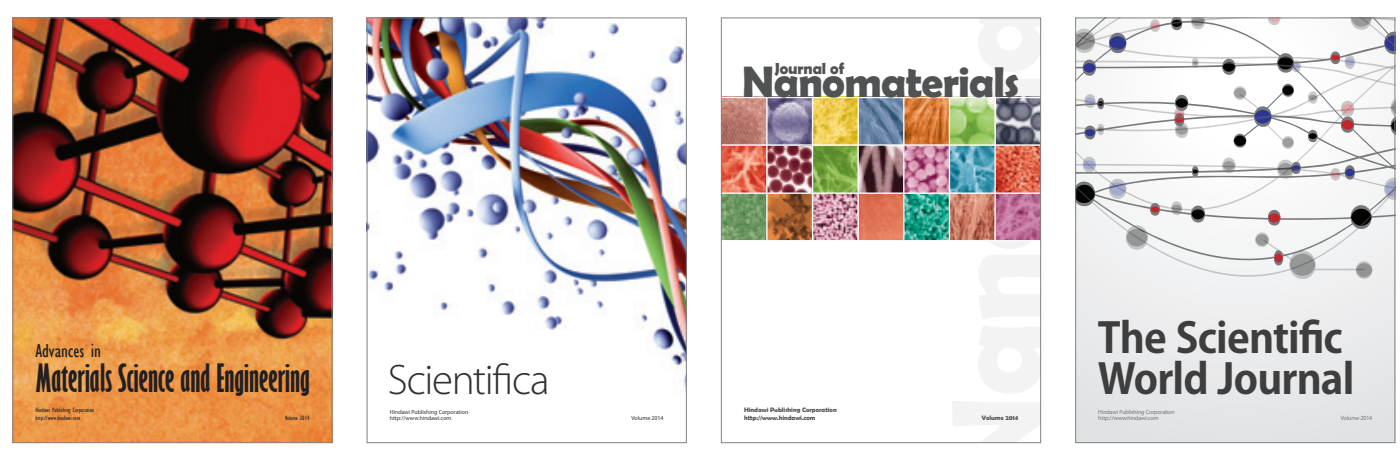

\section{The Scientific World Journal}
